\title{
In-flight Calibration of Hitomi Soft X-ray Spectrometer (3) Effective Area
}

\author{
Masahiro TsuJımoto ${ }^{1}$, Takashi OKAJıMA ${ }^{2}$, Megan E. EсKART' ${ }^{2}$, Takayuki \\ HaYAshi $^{2,3}$, Akio Hoshino ${ }^{2}$, Ryo IIzUKA ${ }^{1}$, Richard L. Kelley ${ }^{2}$, Caroline A. \\ KILbourne $^{2}$, Maurice A. LeutenegGer ${ }^{2,4}$, Yoshitomo MaedA ${ }^{1}$, Hideyuki \\ MORI $^{2}$, Frederick S. PORTER ${ }^{2}$, Kosuke SATO ${ }^{5}$, Toshiki SATO ${ }^{1,6}$, Peter J. \\ SERLEMITSOS ${ }^{2}$, Andrew SzYMKOWIAK ${ }^{7}$, Tahir YAQOOB ${ }^{2,4}$ \\ ${ }^{1}$ Japan Aerospace Exploration Agency, Institute of Space and Astronautical Science, \\ Chuo-ku, Sagamihara, Kanagawa 252-5210, Japan \\ ${ }^{2}$ NASA's Goddard Space Flight Center, Greenbelt, MD 20771, USA \\ ${ }^{3}$ Department of Physics, Nagoya University, Chikusa-ku, Nagoya, Aichi 464-8602, Japan \\ ${ }^{4}$ University of Maryland, Baltimore County, Baltimore, MD 21250, USA \\ ${ }^{5}$ Department of Physics, Tokyo University of Science, Shinjuku-ku, Tokyo 162-8601, Japan \\ ${ }^{6}$ Department of Physics, Tokyo Metropolitan University, Hachioji, Tokyo 192-0397, Japan \\ ${ }^{7}$ Department of Physics, Yale University, New Haven, CT 06520-8120, USA \\ *E-mail: tsujimot@astro.isas.jaxa.jp, takashi.okajima@nasa.gov, megan.e.eckart@nasa.gov
}

Received; Accepted

\begin{abstract}
We present the result of the in-flight calibration of the effective area of the Soft X-ray Spectrometer (SXS) onboard the Hitomi X-ray satellite using an observation of the Crab nebula. We corrected for the artifacts when observing high count rate sources with the X-ray microcalorimeter. We then constructed a spectrum in the $0.5-20 \mathrm{keV}$ band, which we modeled with a single power-law continuum attenuated by an interstellar extinction. We evaluated the systematic uncertainty upon the spectral parameters by various calibration items. In the 2-12 keV band, the SXS result is consistent with the literature values in flux $(2.20 \pm 0.08$ $\times 10^{-8} \mathrm{erg} \mathrm{s}^{-1} \mathrm{~cm}^{-2}$ with a $1 \sigma$ statistical uncertainty) but is softer in the power-law index $(2.19$ \pm 0.11 ). The discrepancy is attributable to the systematic uncertainty of about $+6 /-7 \%$ and $+2 /-5 \%$ respectively for the flux and the power-law index. The softer spectrum is affected primarily by the systematic uncertainty of the Dewar gate valve transmission and the event screening.
\end{abstract}

Key words: Instrumentation: spectrographs — Methods: observational — Space vehicles: instruments

\section{Introduction}

The Soft X-ray Spectrometer (SXS; Kelley et al. 2016) onboard the Hitomi satellite (Takahashi et al. 2016) ceased its short life before it was fully commissioned due to the loss of the spacecraft control. Still, the instrument proved its superb performance, meeting and even partially exceeding the requirements and yielding scientific results. We only have a limited data set during its 38-day operation in the orbit (Tsujimoto et al. 2016), but it added new information that we were unable to obtain during the decade-long preparation on the ground. To make the best use of it, and also to prepare for future X-ray microcalorimeter missions, it is important to verify the instrumental calibration using in-flight data. In this paper, we discuss the effective area calibration of the SXS including its telescope (SXT-S; the soft

(C) 2018. Astronomical Society of Japan. 
X-ray telescope for SXS; Okajima et al. 2016).

The effective area is determined not only by the area of the $\mathrm{X}$-ray mirrors but also by the transmission of various filters, the quantum efficiency and event redistribution of the detector, dead time due to the digital electronics, event screening, the background, the telescope pointing, and the ray-trace modeling. The entire system was not tested end-to-end on the ground. Thus, the total effective area calibration could only be verified with in-flight data. The goal of this paper is to evaluate the inflight observation of a celestial source in light of the available component- and subsystem-level calibration data (table 1).

All ground calibration measurements were performed using the flight unit prior to the launch (Eckart et al. 2016). The only exception is the Dewar gate valve, which was placed at the top of the Dewar to keep the Dewar vacuum on the ground. It was planned to be opened upon confirmation that the initial spacecraft outgassing of potential contaminants ceased $\sim 2$ months after the launch. The spacecraft was lost a few weeks before this operation, thus all data were taken with the gate valve in the optical path. The gate valve window was not fully calibrated on the ground. This choice was motivated by its not being part of the nominal flight configuration as well as schedule considerations. After the loss of the mission, we obtained transmission data on a flight-spare unit manufactured using the same lot of Be as the flight unit (Hoshino et al. 2017; Yoshida et al. 2017), and use these data in this paper.

For this paper, we use the Crab observation simply because we have no other options. It was observed as a commissioning target of other instruments on the spacecraft and was not intended as a calibration source for the SXS. It is a challenging source with a high count rate, but at the same time, we can demonstrate how well we calibrate and model the instrument with small statistical uncertainties.

The Crab is one of the standard candles in the International Astronomical Consortium for High Energy Calibration (IACHEC), and the results of other instruments are available for comparison (Willingale et al. 2001; Weisskopf et al. 2004; Kirsch et al. 2005; Kaastra et al. 2009; Weisskopf et al. 2010; Madsen et al. 2015a). The SXS has a different set of features and systematic uncertainties compared to other X-ray instruments participating in the cross-calibration campaign.

In fact, some features of the SXS are well-suited to observe the Crab nebula. First, it is a non-dispersive spectrometer, so the spectral resolution is not compromised nor complicated by the extended nature of the source. Second, it is a high-resolution spectrometer with a line spread function (LSF) that is dominated by a narrow ( $~ 5 \mathrm{eV}$ FWHM) Gaussian core, and thus the spectrum in the low-energy channels is not very much contaminated by the redistributed events from the high-energy channels. Third, it has a very low non-X-ray background (NXB) at a level of $\lesssim 1$ event per spectral resolution $(5 \mathrm{eV})$ per $100 \mathrm{ks}$ exposure
(Kilbourne et al. 2018). It also achieved a wide high-energy band coverage far beyond the required limit of $12 \mathrm{keV}$. As a result, we obtained a spectrum of the Crab up to $\sim 25 \mathrm{keV}$ above the NXB. Fourth, the sampling rate is much higher $(12.5 \mathrm{kHz})$ than conventional X-ray CCD spectrometers as necessitated for high-resolution microcalorimeter spectroscopy, photon pile-up is much less serious. Fifth, the relative timing accuracy is better than $80 \mu$ s (Leutenegger et al. 2016), which is sufficient to resolve the $34 \mathrm{~ms}$ pulse phases of the Crab pulsar.

A few features of the SXS are not favorable for this source. The first is the coarse spatial resolution of 1.2 half power diameter (Okajima et al. 2016), which is insufficient to spatially resolve the pulsar and the nebula components. We only assess the spatially integrated spectrum within the $3^{\prime}$ square field of view. Second, for high count rate observations like this, we effectively lose exposure time for high spectral resolution events owing to overlapping pulses and overloading of the CPUs in the onboard digital processing unit. The third is the loss of effective area below $\sim 2 \mathrm{keV}$ due to the gate valve Be window. This made it difficult to constrain the amount of interstellar extinction of order $\sim 10^{21.5} \mathrm{~cm}^{-2}$. These features are corrected or evaluated as sources of systematic uncertainties in this paper.

We start with a brief description of the data set $(\S 2)$. We then evaluate the systematic uncertainty by the individual causes (§ 3), and compare them with other results (§ 4). The main results of this study are summarized in $\S 5$. Throughout this paper, we used the HEASoft and CALDB releases on 2017 May 12 for the Hitomi collaboration, the pipeline data products version 03.01.006.007 (Angelini et al. 2016), and the Xspec spectral fitting package version 12.9.1 (Arnaud 1996).

\section{Data}

\subsection{Observation}

The observation was made on 2016 March 25 from 12:35 to 18:01 UT at the position of the Crab pulsar (figure 1). The spacecraft revolved around the Earth 3.6 times and experienced three Earth occultations and three South Atlantic Anomaly (SAA) passages. The total telescope time was $21.5 \mathrm{ks}$, whereas the total on-source time was $9.7 \mathrm{ks}$. Because of the closed gate valve ( $\S 3.4$ ), the effective area below $\sim 2 \mathrm{keV}$ was $0 \mathrm{~cm}^{2}$. The actual incoming rate was $13 \%$ of what we would expect if this source were observed with the open gate valve.

We used cleaned events with an energy registration extended to $32 \mathrm{keV}$. We do not apply the additional screening based on the time proximity of events among different pixels; the false positive by this screening is too large for high count rate observations like this (§3.9). Within the same pixel, events are graded depending on the relative arrival time with the others. In brief, events are graded either into H (high-), M (medium-), or L (lowresolution) for decreasing length to the closest events in time. 
Table 1. References relevant for the SXS and SXT-S effective area calibration

\begin{tabular}{ll}
\hline Item & References \\
\hline Telescope pointing & jaxa_hitomi_memo_2016-001 \\
Mirror effective area & Ground (Iizuka et al. 2014; Sato et al. 2016a; Iizuka et al. JATIS, submitted) and in-flight (Okajima et al. 2016) \\
Mirror edges & Au L (Kikuchi et al. 2016; Maeda et al. 2016) and M edges (Kurashima et al. 2016) \\
Point spread function & Ground (Sato et al. 2014; Iizuka et al. 2014; Sato et al. 2016b; Hayashi et al. 2016) and in-flight (Maeda et al. 2018) \\
Ray-tracing & Yaqoob et al. (JATIS, submitted), asth_sxt_caldb_mirror ${ }^{a}$, asth_sxt_caldb_auxtrans ${ }^{a}$, asth_caldb_telarea ${ }^{a}$ \\
Gate valve & Eckart et al. (2016), Eckart et al. (JATIS, submitted), Hoshino et al. (2017); Yoshida et al. (2017), asth_sxs_caldb_gatevalve ${ }^{a}$ \\
Aperture filters & Eckart et al. (2016); Kilbourne et al. (2016a), Eckart et al. (JATIS, submitted), asth_sxs_caldb_blckfilt ${ }^{a}$ \\
Quantum efficiency & Eckart et al. (2016), Eckart et al. (JATIS, submitted), asth_sxs_caldb_quanteff ${ }^{a}$ \\
Energy gain & Eckart et al. (2016); Leutenegger et al. (2016), Eckart et al. (JATIS, submitted), asth_sxs_caldb_gainpix ${ }^{a}$ \\
Line spread function & Eckart et al. (2016); Leutenegger et al. (2016), asth_sxs_caldb_rmfparam ${ }^{a}$ \\
Data processing & Ishisaki et al. (2016); Angelini et al. (2016) \\
Time assignment & Eckart et al. (2016), asth_sxs_caldb_coeftime \\
NXB & Kilbourne et al. (2016b); Porter et al. (2016); Kilbourne et al. (2018) \\
\hline
\end{tabular}

${ }^{a}$ Available at https://heasarc.gsfc.nasa.gov/docs/hitomi/calib/hitomi_caldb_docs.html.

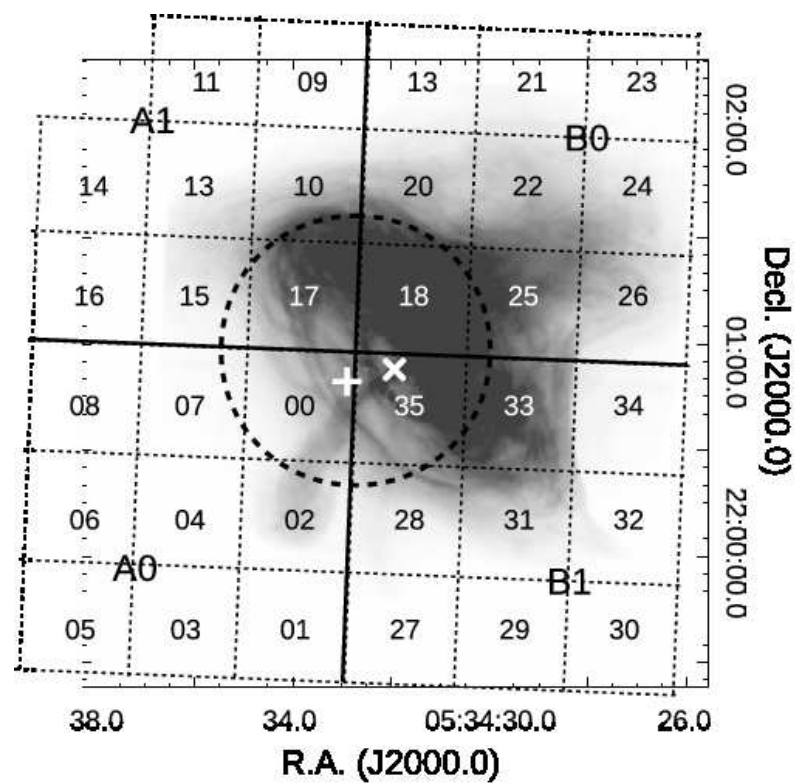

Fig. 1. Field of view of the four quadrants $(A 0, A 1, B 0$, and $B 1)$ that comprises a $6 \times 6$ array (dotted squares with pixel numbers) superimposed on the Chandra ACIS image after correcting for the readout streaks (Mori et al. 2004). The top left pixel in the array is not read out to accommodate a dedicated calibration pixel (number 12) that does not receive celestial photons. A half power diameter circle of the SXS is shown around the array center with the dashed circle. The position of the optical axis and the pulsar (Lobanov et al. 2011) are respectively shown with the plus and cross signs.

They are also graded either $\mathrm{p}$ (primary) or s (secondary) if it is the leading pulse or not. When an event is not seriously overlapped by other events close in time, the accuracy of the energy determination is high enough for high-resolution spectroscopy. The Hp and Mp grades are recognized as such, which we call "high-quality" grades. The total number of cleaned events is $1.8 \times 10^{6}$ with an average count rate of $5.3 \mathrm{~s}^{-1}$ pixel $^{-1}$. About $43 \%$ of the events are of a high-quality grade. We use events of all grades for a better statistics in this paper, as we are mostly interested in the overall spectral shape.

\subsection{Time series}

The telescope pointing was measured and corrected using the star tracker (STT) and the inertial reference unit. The STT achieves a better accuracy but was not in use during the Earth occultations or SAA passages. The actual pointing fluctuation was measured by calculating the median values of the detector coordinate of all X-ray events in $30 \mathrm{~s}$ time slices during the on-source time (figure 2a). Some jumps in the coordinate (e.g., 11.7 and $17.5 \mathrm{ks}$ from the start) were seen when the STT service was started and the pointing was quickly tuned. The degradation of the control is found when STT was unavailable (e.g., 7.3-8.2 ks). When the STT was used, the pointing was accurate to $0 .{ }^{\prime} 2$ and was stable to $2 .^{\prime \prime} 9$ at a $1 \sigma$ level. When the STT was not used, the pointing was accurate to $3 .{ }^{\prime \prime} 3$ and was stable to $4 . \prime 0$ at $1 \sigma$. These fluctuations are small enough in comparison to the detector pixel scale of $30^{\prime \prime}$, but it left a clear signature in the X-ray count rate from a source that would otherwise be stable on a time scale of the observation.

The total throughput of the SXS for bright sources is determined by the CPU processing speed of the onboard digital electronics called the Pulse Shape Processor (PSP; Ishisaki et al. 2016). The PSP consists of two identical units (PSP-A and PSPB), and each unit has one FPGA and two CPU boards. A total of 36 pixels is processed independently with no priority for a particular pixel. All cross-channel processes including the anticoincidence (anti-co) veto are performed in the ground processing. A quarter of the FPGA and CPU resources (called A0, A1, $\mathrm{B} 0$, and B1) handle nine pixels in a quadrant of the array (figure 1). Pixel 12 is offset from the array and is illuminated by an ${ }^{55} \mathrm{Fe}$ calibration source with a constant rate of $3.9 \mathrm{~s}^{-1}$, which is processed with the A1 quadrant.

The FPGA board detects event candidates in the continuously input time series. Their statistics are included in the house-keeping telemetry. The CPU board searches for overlapping events upon the FPGA-detected event candidates, de- 

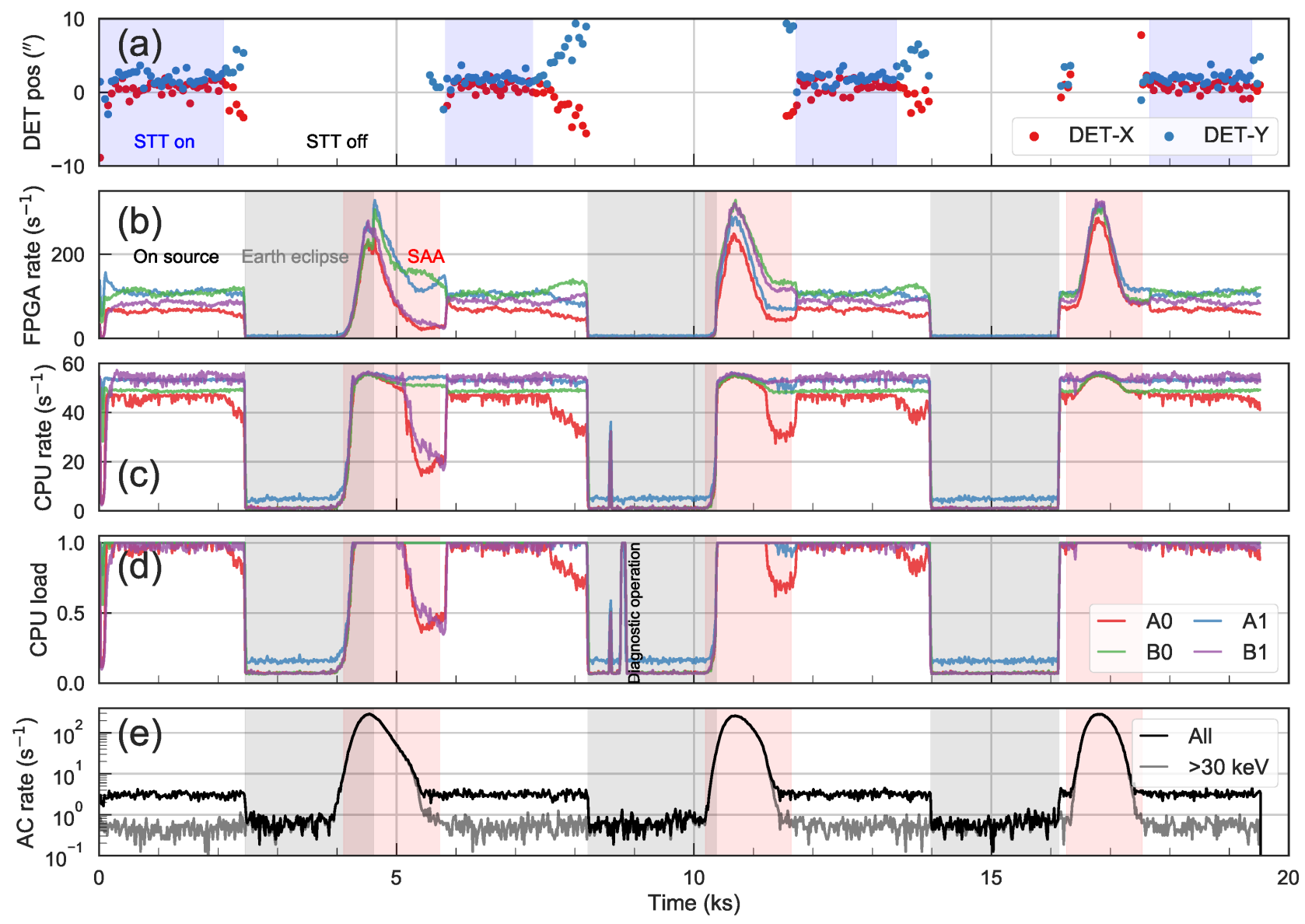

Fig. 2. Time series of the (a) median values of the detector coordinates of all events in a $30 \mathrm{~s}$ interval, (b) calorimeter event candidate rate detected by the FPGA, (c) calorimeter event rate processed by the CPU, (d) CPU load, and (e) anti-co event rate for all events (black) and those used for veto screening with energies above $30 \mathrm{keV}$ (gray). The panels (b)-(d) are shown for the sum of all pixels in each of the four quadrants. Gray and red shades respectively indicate the duration of Earth eclipse and the SAA passages. The remainder is the on-source time. A part of it was operated with the STT, which is shown in the blue shade in panel (a). The spikes in (c) and (d) in 8-9 ks are due to diagnostic operations. The start of the observation is 2016/03/25 12:35:48 UT.

blends them, and derives the arrival time and the energy by optimal filtering for each event, and assign flags and grades to them. All these events are recorded in the event telemetry. The FPGA and CPU rates are shown respectively in figure 2 (b) and (c). The FPGA has a sufficient buffer size for this observation, thus the rate is a proxy for the actual incoming rate. On the other hand, the CPU speed was not fast enough and all four CPUs were fully loaded during most of the Crab observation when the FPGA rate exceeded $\sim 50 \mathrm{~s}^{-1}$ per quadrant, as shown in figure 2 (d).

At full load, the entire data buffer of up to 256 events stored by the FPGA is occasionally discarded to catch up, which causes effective dead time. This is executed pixel by pixel with no preference for a particular pixel, and lost times are recorded. We call this "pixel dead time" and the fraction of its complement as "live time fraction", hereafter. As a result, the rate of CPU-processed events (figure 2c) is a fraction of the actual incoming rate. The anti-co events are detected by the FPGA and are not processed by the CPU, thus have no loss of events dur- ing the observation (figure 2e). High-energy events of the Crab were detected by the anti-co, which is recognized by the elevated count rate during the on-source time in comparison to the Earth eclipses for all events (black curve in figure 2e). This discontinuity is not seen for anti-co events for those with energies above $30 \mathrm{keV}$ (gray curve), which are used for veto screening.

\subsection{Image}

Figure 3 shows the count rate map of (a) the event candidates detected by the FPGA, (b) the events processed by the CPU, (c) the events of the high-quality grades, and (d) the live time fraction during the on-source time. This illustrates various artifacts when observing a bright source with the SXS. Because of the pixel dead time, more illuminated pixels suffer a larger loss of events, thus the live time fraction is lower at the center of the array (figure $3 \mathrm{~d}$ ). The map of events actually processed by the CPU (figure $3 b$ ) should be corrected for this to derive the map of actual rate incident on the detector (figure $3 \mathrm{e}$ ). The event 


\begin{tabular}{|c|c|c|c|c|c|c|}
\hline \multirow[t]{2}{*}{ (a) } & $\begin{array}{l}\text { P23 } \\
2.03\end{array}$ & $\begin{array}{l}\text { P24 } \\
3.69\end{array}$ & $\begin{array}{l}\text { P26 } \\
4.52\end{array}$ & $\begin{array}{l}\text { P34 } \\
3.23\end{array}$ & $\begin{array}{l}\text { P32 } \\
2.25\end{array}$ & $\begin{array}{l}\text { P30 } \\
1.29\end{array}$ \\
\hline & $\begin{array}{l}\text { P21 } \\
3.87\end{array}$ & $\begin{array}{c}\text { P22 } \\
10.70\end{array}$ & $\begin{array}{c}\text { P25 } \\
16.01\end{array}$ & $\begin{array}{c}\text { P33 } \\
16.44\end{array}$ & $\begin{array}{l}\text { P31 } \\
8.00\end{array}$ & $\begin{array}{l}\text { P29 } \\
2.83\end{array}$ \\
\hline $\begin{array}{l}\text { acB } \\
0.17\end{array}$ & $\begin{array}{l}\text { P19 } \\
5.78\end{array}$ & $\begin{array}{c}\text { P20 } \\
18.73\end{array}$ & $\begin{array}{c}\text { P18 } \\
37.35\end{array}$ & $\begin{array}{c}\text { P35 } \\
34.39\end{array}$ & $\begin{array}{c}\text { P28 } \\
13.69\end{array}$ & $\begin{array}{c}\text { P27 } \\
35.60\end{array}$ \\
\hline \multirow[t]{3}{*}{$\begin{array}{l}\text { acA } \\
0.20\end{array}$} & $\begin{array}{c}\text { P9 } \\
4.75\end{array}$ & $\begin{array}{c}\text { P10 } \\
21.32\end{array}$ & $\begin{array}{c}\text { P17 } \\
35.60\end{array}$ & $\begin{array}{c}\text { P0 } \\
27.49\end{array}$ & $\begin{array}{c}\text { P2 } \\
11.47\end{array}$ & $\begin{array}{c}\text { P1 } \\
4.20\end{array}$ \\
\hline & $\begin{array}{l}\text { P11 } \\
3.90\end{array}$ & $\begin{array}{c}\text { P13 } \\
11.98\end{array}$ & $\begin{array}{c}\text { P15 } \\
15.62\end{array}$ & $\begin{array}{c}P 7 \\
10.73\end{array}$ & $\begin{array}{c}\text { P4 } \\
5.97\end{array}$ & $\begin{array}{c}\text { P3 } \\
2.32\end{array}$ \\
\hline & & $\begin{array}{l}\text { P14 } \\
3.57\end{array}$ & $\begin{array}{l}\text { P16 } \\
3.98\end{array}$ & $\begin{array}{c}\text { P8 } \\
3.60\end{array}$ & $\begin{array}{c}\mathrm{P} 6 \\
2.22\end{array}$ & $\begin{array}{c}\text { P5 } \\
1.02\end{array}$ \\
\hline $\begin{array}{l}\mathrm{P} 12 \\
5.98\end{array}$ & & & & & & \\
\hline
\end{tabular}

\begin{tabular}{|c|c|c|c|c|c|c|}
\hline (b) & $\begin{array}{l}\text { P23 } \\
1.41\end{array}$ & $\begin{array}{l}\text { P24 } \\
2.60\end{array}$ & $\begin{array}{l}\text { P26 } \\
2.79\end{array}$ & $\begin{array}{l}\text { P34 } \\
2.21\end{array}$ & $\begin{array}{l}\text { P32 } \\
1.56\end{array}$ & $\begin{array}{l}\text { P30 } \\
0.96\end{array}$ \\
\hline & $\begin{array}{l}\text { P21 } \\
2.74\end{array}$ & $\begin{array}{l}\text { P22 } \\
7.80\end{array}$ & $\begin{array}{l}\text { P25 } \\
9.22\end{array}$ & $\begin{array}{c}\text { P33 } \\
11.30\end{array}$ & $\begin{array}{l}\text { P31 } \\
5.58\end{array}$ & $\begin{array}{l}\text { P29 } \\
1.93\end{array}$ \\
\hline $\begin{array}{l}\mathrm{acB} \\
0.17\end{array}$ & $\begin{array}{l}\text { P19 } \\
3.85\end{array}$ & $\begin{array}{l}\text { P20 } \\
9.21\end{array}$ & $\begin{array}{l}\text { P18 } \\
8.67\end{array}$ & $\begin{array}{c}\text { P35 } \\
17.45\end{array}$ & $\begin{array}{l}\text { P28 } \\
9.80\end{array}$ & $\begin{array}{l}\text { P27 } \\
9.61\end{array}$ \\
\hline \multirow[t]{3}{*}{$\begin{array}{l}\text { acA } \\
0.20\end{array}$} & $\begin{array}{c}\text { P9 } \\
3.32\end{array}$ & $\begin{array}{l}\text { P10 } \\
9.87\end{array}$ & $\begin{array}{l}\text { P17 } \\
9.61\end{array}$ & $\begin{array}{c}\text { P0 } \\
17.02\end{array}$ & $\begin{array}{c}\text { P2 } \\
8.13\end{array}$ & $\begin{array}{c}\mathrm{P} 1 \\
2.83\end{array}$ \\
\hline & $\begin{array}{l}\mathrm{P} 11 \\
2.71\end{array}$ & $\begin{array}{l}\mathrm{P} 13 \\
8.35\end{array}$ & $\begin{array}{l}\text { P15 } \\
9.95\end{array}$ & $\begin{array}{c}\text { P7 } \\
7.75\end{array}$ & $\begin{array}{c}P 4 \\
4.14\end{array}$ & $\begin{array}{c}\text { P3 } \\
1.59\end{array}$ \\
\hline & & $\begin{array}{l}\text { P14 } \\
2.43\end{array}$ & $\begin{array}{l}\text { P16 } \\
2.71\end{array}$ & $\begin{array}{c}\text { P8 } \\
2.41\end{array}$ & $\begin{array}{c}\text { P6 } \\
1.51\end{array}$ & $\begin{array}{c}\text { P5 } \\
0.71\end{array}$ \\
\hline $\begin{array}{l}\mathrm{P} 12 \\
3.78\end{array}$ & & & & & & \\
\hline
\end{tabular}

\begin{tabular}{|c|c|c|c|c|c|c|}
\hline (c) & $\begin{array}{l}\text { P23 } \\
1.26\end{array}$ & $\begin{array}{l}\text { P24 } \\
2.06\end{array}$ & $\begin{array}{l}\text { P26 } \\
2.17\end{array}$ & $\begin{array}{l}\text { P34 } \\
1.81\end{array}$ & $\begin{array}{l}\text { P32 } \\
1.32\end{array}$ & $\begin{array}{l}\text { P30 } \\
0.86\end{array}$ \\
\hline & $\begin{array}{l}\text { P21 } \\
2.11\end{array}$ & $\begin{array}{l}\text { P22 } \\
3.94\end{array}$ & $\begin{array}{l}\text { P25 } \\
3.25\end{array}$ & $\begin{array}{l}\text { P33 } \\
4.17\end{array}$ & $\begin{array}{l}\text { P31 } \\
3.42\end{array}$ & $\begin{array}{l}\text { P29 } \\
1.61\end{array}$ \\
\hline $\begin{array}{l}\text { acB } \\
0.17\end{array}$ & $\begin{array}{l}\text { P19 } \\
2.76\end{array}$ & $\begin{array}{l}\text { P20 } \\
2.78\end{array}$ & $\begin{array}{l}\text { P18 } \\
0.55\end{array}$ & $\begin{array}{l}\text { P35 } \\
1.57\end{array}$ & $\begin{array}{l}\text { P28 } \\
4.12\end{array}$ & $\begin{array}{l}\text { P27 } \\
0.75\end{array}$ \\
\hline \multirow[t]{3}{*}{$\begin{array}{l}\text { acA } \\
0.20\end{array}$} & $\begin{array}{c}\text { P9 } \\
2.49\end{array}$ & $\begin{array}{l}\text { P10 } \\
2.41\end{array}$ & $\begin{array}{l}\text { P17 } \\
0.75\end{array}$ & $\begin{array}{c}\mathrm{PO} \\
2.56\end{array}$ & $\begin{array}{c}\text { P2 } \\
3.96\end{array}$ & $\begin{array}{c}\text { P1 } \\
2.18\end{array}$ \\
\hline & $\begin{array}{l}\text { P11 } \\
2.11\end{array}$ & $\begin{array}{l}\text { P13 } \\
3.94\end{array}$ & $\begin{array}{l}\text { P15 } \\
3.62\end{array}$ & $\begin{array}{c}\text { P7 } \\
3.96\end{array}$ & $\begin{array}{c}P 4 \\
2.86\end{array}$ & $\begin{array}{c}\text { P3 } \\
1.40\end{array}$ \\
\hline & & $\begin{array}{l}\text { P14 } \\
1.95\end{array}$ & $\begin{array}{l}\text { P16 } \\
2.14\end{array}$ & $\begin{array}{c}\text { P8 } \\
1.94\end{array}$ & $\begin{array}{c}\text { P6 } \\
1.35\end{array}$ & $\begin{array}{c}\text { P5 } \\
0.67\end{array}$ \\
\hline $\begin{array}{l}\mathrm{P} 12 \\
2.70\end{array}$ & & & & & & \\
\hline
\end{tabular}

\begin{tabular}{|c|c|c|c|c|c|c|}
\hline (d) & $\begin{array}{l}\text { P23 } \\
1.00\end{array}$ & $\begin{array}{l}\text { P24 } \\
1.00\end{array}$ & $\begin{array}{l}\text { P26 } \\
1.00\end{array}$ & $\begin{array}{l}\text { P34 } \\
1.00\end{array}$ & $\begin{array}{l}\text { P32 } \\
1.00\end{array}$ & $\begin{array}{l}\text { P30 } \\
1.00\end{array}$ \\
\hline & $\begin{array}{l}\text { P21 } \\
1.00\end{array}$ & $\begin{array}{l}\text { P22 } \\
0.94\end{array}$ & $\begin{array}{l}\mathrm{P} 25 \\
0.72\end{array}$ & $\begin{array}{l}\text { P33 } \\
0.98\end{array}$ & $\begin{array}{l}\text { P31 } \\
1.00\end{array}$ & $\begin{array}{l}\text { P29 } \\
1.00\end{array}$ \\
\hline $\begin{array}{l}\mathrm{acB} \\
1.00\end{array}$ & $\begin{array}{l}\text { P19 } \\
0.99\end{array}$ & $\begin{array}{l}\text { P20 } \\
0.61\end{array}$ & $\begin{array}{l}\mathrm{P} 18 \\
0.27\end{array}$ & $\begin{array}{l}\text { P35 } \\
0.66\end{array}$ & $\begin{array}{l}\text { P28 } \\
1.00\end{array}$ & $\begin{array}{l}\text { P27 } \\
0.37\end{array}$ \\
\hline \multirow[t]{3}{*}{$\begin{array}{l}\text { acA } \\
1.00\end{array}$} & $\begin{array}{c}\text { P9 } \\
0.98\end{array}$ & $\begin{array}{l}\text { P10 } \\
0.62\end{array}$ & $\begin{array}{l}\text { P17 } \\
0.37\end{array}$ & $\begin{array}{c}\mathrm{P} 0 \\
0.89\end{array}$ & $\begin{array}{c}\mathrm{P} 2 \\
1.00\end{array}$ & $\begin{array}{c}\mathrm{P} 1 \\
1.00\end{array}$ \\
\hline & $\begin{array}{l}\text { P11 } \\
0.99\end{array}$ & $\begin{array}{l}\mathrm{P} 13 \\
0.99\end{array}$ & $\begin{array}{l}\mathrm{P} 15 \\
0.92\end{array}$ & $\begin{array}{c}\text { P7 } \\
1.00\end{array}$ & $\begin{array}{c}\mathrm{P} 4 \\
1.00\end{array}$ & $\begin{array}{c}\text { P3 } \\
1.00\end{array}$ \\
\hline & & $\begin{array}{l}\text { P14 } \\
1.00\end{array}$ & $\begin{array}{l}\text { P16 } \\
1.00\end{array}$ & $\begin{array}{c}\text { P8 } \\
1.00\end{array}$ & $\begin{array}{c}\text { P6 } \\
1.00\end{array}$ & $\begin{array}{c}\text { P5 } \\
1.00\end{array}$ \\
\hline $\begin{array}{l}\mathrm{P} 12 \\
1.00\end{array}$ & & & & & & \\
\hline
\end{tabular}

\begin{tabular}{|c|c|c|c|c|c|c|}
\hline$(\mathrm{e})$ & $\mathrm{P} 23$ & $\mathrm{P} 24$ & $\mathrm{P} 26$ & $\mathrm{P} 34$ & $\mathrm{P} 32$ & $\mathrm{P} 30$ \\
& 1.41 & 2.60 & 2.79 & 2.21 & 1.56 & 0.96 \\
& $\mathrm{P} 21$ & $\mathrm{P} 22$ & $\mathrm{P} 25$ & $\mathrm{P} 33$ & $\mathrm{P} 31$ & $\mathrm{P} 29$ \\
& 2.75 & 8.27 & 12.79 & 11.55 & 5.58 & 1.93 \\
\hline acB & $\mathrm{P} 19$ & $\mathrm{P} 20$ & $\mathrm{P} 18$ & $\mathrm{P} 35$ & $\mathrm{P} 28$ & $\mathrm{P} 27$ \\
0.17 & 3.90 & 15.18 & 32.23 & 26.31 & 9.80 & 26.05 \\
\hdashline acA & $\mathrm{P9}$ & $\mathrm{P} 10$ & $\mathrm{P} 17$ & $\mathrm{P} 0$ & $\mathrm{P} 2$ & $\mathrm{P} 1$ \\
0.20 & 3.39 & 15.95 & 26.05 & 19.22 & 8.13 & 2.83 \\
& & & & & & \\
& $\mathrm{P} 11$ & $\mathrm{P} 13$ & $\mathrm{P} 15$ & $\mathrm{P} 7$ & $\mathrm{P} 4$ & $\mathrm{P} 3$ \\
& 2.73 & 8.47 & 10.86 & 7.75 & 4.14 & 1.59 \\
& & $\mathrm{P} 14$ & $\mathrm{P} 16$ & $\mathrm{P} 8$ & $\mathrm{P} 6$ & $\mathrm{P} 5$ \\
& & 2.43 & 2.71 & 2.41 & 1.51 & 0.71 \\
\hline $\mathrm{P} 12$ & & & & & & \\
\hline 3.78 & & & & & & \\
\hline
\end{tabular}

Fig. 3. Count rate map in the unit of $\mathrm{s}^{-1}$ of (a) event candidates detected by the FPGA, (b) events processed by the CPU not corrected for the pixel dead time, and (c) the events of a high-quality grade during on-source time, which is an average of the duration of 18-19 ks from the start of the observation (figure 2). (d) is the live time fraction of all on-source time. (e) processed event rate corrected for the pixel dead time by calculating (b) divided by (d). The maps are in the detector coordinate, as opposed to figure 1 in the sky coordinate. The positions of the anti-co (acA and acB) and calibration ( $\mathrm{P} 12)$ channels are arbitrary. 
candidate rate by FPGA (figure 3a) does not exactly match with figure $3 \mathrm{e}$, as figure $3 \mathrm{a}$ is a rate of candidates without inspecting the shapes of individual pulses. When the incoming rate is too high, the rate of high-quality grades decreases. When all these are combined, the map of the high-quality grade events has a ring-like structure (figure $3 \mathrm{c}$ ).

\subsection{Spectrum}

Figure 4 (a) shows the source and NXB spectra. The source spectrum was integrated from the entire field of view and averaged over the pulse phases. The NXB dominates the background of the SXS. The source has an excess signal against the $\mathrm{NXB}$ up to $\sim 25 \mathrm{keV}$. As the effective area drops sharply below $\sim 2 \mathrm{keV}$, events below the energy are mostly redistributed events from high-energy channels.

We compared the NXB-subtracted spectrum with a spectral model of the Crab nebula. The Crab X-ray emission mainly consists of (i) the point-like, pulsed, and harder emission from the pulsar and (ii) the extended, unpulsed, and softer emission from the synchrotron nebula. However, the spectrum integrated over the space and time can be simply described by a power-law of an index 2.1 without a break for the XMM-Newton EPICpn (0.7-10 keV), RXTE PCA (3-60 keV), and NuSTAR (3$78 \mathrm{keV}$ ) spectra (Weisskopf et al. 2010; Shaposhnikov et al. 2012; Madsen et al. 2015b), so we used a single power-law continuum attenuated by an interstellar extinction.

For the power-law component, we used the pegpwrlw model, in which the unabsorbed flux in a given range is a free parameter. The flux is much better decoupled with the powerlaw index than the conventional power-law model, in which the intensity at $1 \mathrm{keV}$ is a free parameter. For the extinction model, we used the tbabs model version 2.3.2 ${ }^{1}$ (Wilms et al. 2000). As we have no sensitivity below $\sim 2 \mathrm{keV}$ due to the closed gate valve, we cannot constrain the extinction column $\left(N_{\mathrm{H}}\right)$. We thus fixed this parameter to be $4.2 \times 10^{21} \mathrm{~cm}^{-2}$ with the oxygen abundance decreased to 0.676 (Weisskopf et al. 2004) relative to solar (Wilms et al. 2000).

We call this model the canonical model, in which the flux is the only free parameter. We convolved the model with the instrumental response and fitted it to the data. The best-fit parameters are shown in table 2, while the ratio of the data against the model is shown in figure 4 (b). The auxiliary response function (ARF) and the redistribution matrix function (RMF) were generated following the data analysis guide ${ }^{2}$. In the calculation of ARF, we considered the extended structure of the Crab nebula using the X-ray image taken with the Chandra ACIS (Mori et al. 2004) and the offset position of the optical axis from the

\footnotetext{
${ }^{1}$ See http://pulsar.sternwarte.uni-erlangen.de/wilms/research/ tbabs/ for details.

${ }^{2}$ The document is available at https://heasarc.gsfc.nasa.gov/docs/ hitomi/analysis/.
}

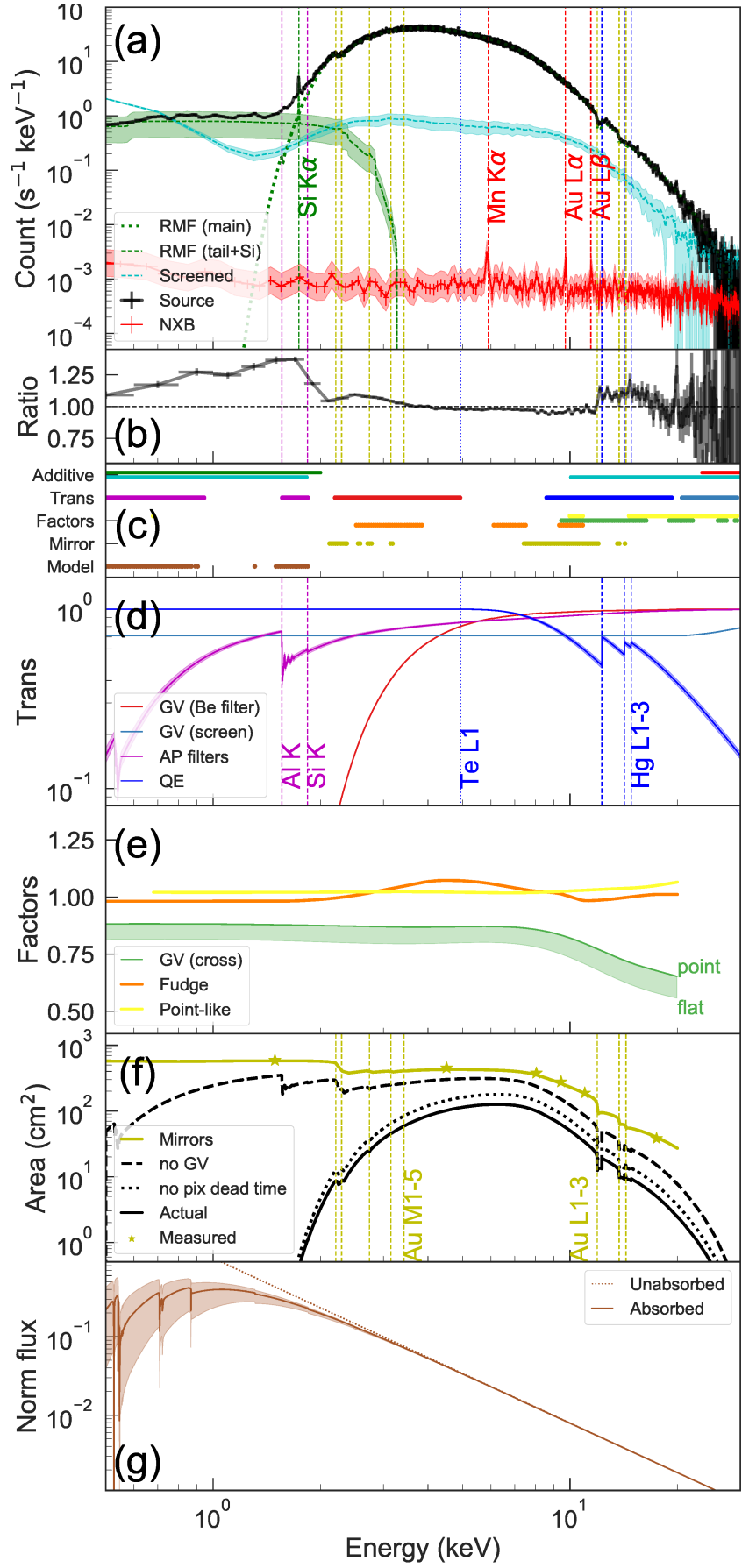

Fig. 4. (a) Source (black) spectrum and its decomposition into the main portion of the response (dominated by Gaussian core; dotted green) and the redistribution tail (dashed green), as well as the NXB spectrum (red) and the spectrum of the screened events (cyan), are shown. Instrumental features are labeled with the color corresponding to the relevant part of the spectrum. (b) Ratio of the background-subtracted spectrum and the canonical model (table 2) of the Crab nebula. (c) Energy band in which the individual causes are important. (d) Transmission of the gate valve Be filter and the support mesh structure, the aperture filters, and the quantum efficiency of the detector. (e) Correction function of the gate valve support cross structure, the SXT-S fudge function, and the extended nature of the source. ( $f$ ) Effective area of the mirror assembly with the six measurements on the ground (yellow; lizuka et al. JATIS, submitted) and the actual effective area (black solid) as well as that expected without the gate valve (dashed black) and without the pixel dead time (dotted black). (g) Spectral model. Both the unabsorbed and the absorbed power-law models are shown. The shaded range indicates the adopted uncertainty of the absorption. 


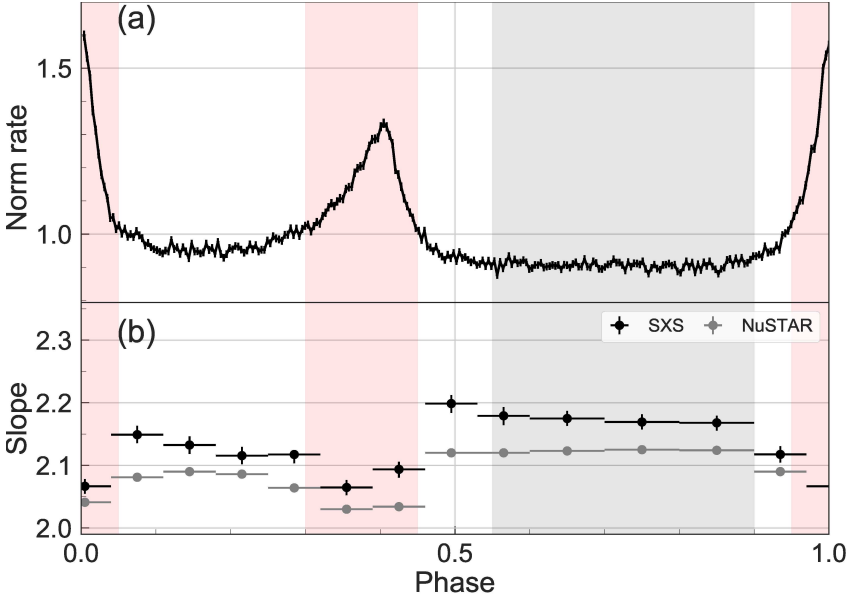

Fig. 5. (a) Folded light curve using events in the $2-20 \mathrm{keV}$ band. Red and black shades define the on- and off-pulse phases, each of which occupies $35 \%$ of a cycle. (b) Best-fit and $1 \sigma$ statistical uncertainty of the power-law index for phase-sliced spectroscopy of the SXS and NuSTAR (Madsen et al. 2015b).

array center and the Crab pulsar position (figure 1). The redistribution into the $\mathrm{Si} \mathrm{K}$ fluorescence is not included in the RMF generator ( $\S 3.7$ ), so we added two Lorentzian models to represent the $\mathrm{Si} \mathrm{K} \alpha_{1}$ and $\mathrm{K} \alpha_{2}$ lines.

Table 2. Canonical model parameters in $0.5-24 \mathrm{keV}$.

\begin{tabular}{lll}
\hline Component & Parameter & Value $^{b}$ \\
\hline Power-law & Index & 2.1 \\
& Flux & $(4.947 \pm 0.037) \times 10^{-8} \mathrm{erg} \mathrm{s}^{-1} \mathrm{~cm}^{-2}$ \\
Extinction & $N_{\mathrm{H}}$ & $4.2 \times 10^{21} \mathrm{~cm}^{-2}$ \\
& O abundance & $0.676 \mathrm{solar}$ \\
& Other abundance & 1.0 solar \\
\hline $\mathrm{Si} \mathrm{K} \alpha^{a}$ & $\mathrm{~K} \alpha_{1}$ energy & $1.73998 \mathrm{keV}$ \\
& $\mathrm{K} \alpha_{2}$ energy & $1.73939 \mathrm{keV}$ \\
& Gain offset & $-2.66_{-0.29}^{+0.33} \mathrm{eV}$ \\
& $\mathrm{K} \alpha_{1}$ intensity & $5.3_{-0.4}^{+0.7} \times 10^{-2} \mathrm{~cm}^{-2} \mathrm{~s}^{-1}$ \\
& FWHM & $1.3_{-0.75}^{+1.4} \mathrm{eV}$ \\
\hline Fit goodness & Reduced $\chi^{2} / \mathrm{dof}$ & $1.12 / 23498$ \\
\hline
\end{tabular}

${ }^{a}$ Two Lorentzian models were used to describe the natural line shape of $\mathrm{Si} \mathrm{K} \alpha_{1}$ and $\mathrm{K} \alpha_{2}$. The two energies were allowed to shift collectively to adjust for the known SXS gain offset (Eckart et al. 2016). The $\mathrm{K} \alpha_{2}$ intensity was fixed at half of that of $\mathrm{K} \alpha_{1}$. These parameters were derived in the local fitting in $1.7-1.78 \mathrm{keV}$, then the best-fit values were used in the broadband fittings.

${ }^{b}$ Errors indicate a $1 \sigma$ statistical uncertainty. Those without errors are fixed values.

\subsection{Pulse Phase}

Figure 5 (a) shows the light curve folded by the pulse period after a barycentric correction for the position (RA, Dec) = $\left(05^{\mathrm{h}} 34^{\mathrm{m}} 31^{\mathrm{s}} .97232,+22^{\circ} 00^{\prime} 52^{\prime \prime}\right.$.069) in the equinox J2000.0. Events with all grades in the $2-20 \mathrm{keV}$ band were used. The pulse period and its time derivative were derived as $33.7204626 \mathrm{~ms}$ and $4.198 \times 10^{-13} \mathrm{~s} \mathrm{~s}^{-1}$ using the present data. The ephemeris was determined based on simultaneous radio ob- servations (Hitomi Collaboration 2018). The SXS events are assigned times based on a clock synchronized to an onboard Global Positioning System receiver. The accuracy of these time assignments is better than $20 \mu$ s (Leutenegger et al. 2016). The durations of the pixel dead times are longer than $5 \mathrm{~s}$. Therefore, the $34 \mathrm{~ms}$ pulse profile is little distorted by the dead times.

\section{Analysis}

In this section, we evaluate the systematic uncertainties by various causes in the SXS spectral fitting. Looking at the comparison between the data and the canonical model (figure $4 \mathrm{~b}$ ), we find various deviations in the different energy bands, which suggests that different causes of systematic uncertainties dominate in the different bands. We divided the energy band into three: 2-4, 4-12, and 12-24 keV. In addition, we evaluated in the energy band extended to the soft energies $(0.5-12 \mathrm{keV})$, a part of it limited by the gate valve (2-12 keV), and the entire band including the extended parts on both soft and hard band $(0.5-24 \mathrm{keV})$.

For each energy band, we fit the spectrum with the fiducial model, in which the photon index was also treated as a free parameter in the canonical model. We evaluate the effect of each individual source of systematic uncertainties $(\S 3.1-\S 3.11)$ in terms of the best-fit values of the free parameters (photon index and the flux) in the fiducial model. Different values of $N_{\mathrm{H}}$ are considered as a source of systematic uncertainties ( $\S 3.11$ ). The result is summarized in each of the lines in table 3 , while their effects are graphically shown in the panels in figure 4.

\subsection{Telescope pointing}

When the telescope optical axis is pointed off of a point-like source, a larger fraction of photons land outside of the field of view, and thus the effective area decreases. This is further complicated by the distribution of the pixel dead time (figure $3 \mathrm{~d}$ ). Based on the telescope pointing accuracy (figure 2a), we evaluated this effect by placing the optical axis position at offset places when calculating the effective area.

\subsection{Image extent}

The effective area is calculated based on the Chandra image (figure 1). In order to assess the systematic uncertainty by this assumption, we generated an ARF assuming that the Crab nebula is a point-like source. The ratio of the effective area curve assuming a point-like source with respect to that assuming the Chandra image (figure 1) is shown in figure 4 (e). 
Table 3. Statistical and systematic uncertainties of the fiducial model fitting.

\begin{tabular}{|c|c|c|c|c|c|c|c|c|c|c|c|c|}
\hline \multirow{2}{*}{$\begin{array}{l}\text { Parameter } \\
\text { Energy band }(\mathrm{keV})\end{array}$} & \multirow[b]{2}{*}{$2-4$} & \multicolumn{4}{|c|}{- Flux $^{a}$} & \multirow[b]{2}{*}{$0.5-24$} & \multirow[b]{2}{*}{$2-4$} & \multicolumn{4}{|c|}{ Power-law index } & \multirow[b]{2}{*}{$0.5-24$} \\
\hline & & $4-12$ & $12-24$ & $0.5-12$ & $2-12$ & & & $4-12$ & $12-24$ & $0.5-12$ & $2-12$ & \\
\hline Best-fit $^{b}$ & 0.95 & 1.26 & 0.80 & 4.52 & 2.20 & 5.15 & 2.26 & 2.13 & 2.28 & 2.19 & 2.19 & 2.18 \\
\hline Statistical $(\%)^{c}$ & \pm 0.17 & \pm 0.11 & \pm 1.04 & \pm 0.18 & \pm 0.08 & \pm 0.13 & \pm 0.44 & \pm 0.17 & \pm 2.10 & \pm 0.09 & \pm 0.11 & \pm 0.09 \\
\hline Systematic $(\%)^{d}$ & $\begin{array}{l}+7.06 \\
-13.1 \\
\end{array}$ & $\begin{array}{l}+6.26 \\
-4.23 \\
\end{array}$ & $\begin{array}{l}+22.1 \\
-9.22 \\
\end{array}$ & $\begin{array}{l}+6.51 \\
-13.2 \\
\end{array}$ & $\begin{array}{l}+5.74 \\
-6.55 \\
\end{array}$ & $\begin{array}{l}+6.00 \\
-10.5 \\
\end{array}$ & $\begin{array}{l}+6.74 \\
-11.8 \\
\end{array}$ & $\begin{array}{l}+4.58 \\
-4.25 \\
\end{array}$ & $\begin{array}{l}+8.27 \\
-5.79 \\
\end{array}$ & $\begin{array}{l}+1.94 \\
-4.49 \\
\end{array}$ & $\begin{array}{l}+2.09 \\
-4.63 \\
\end{array}$ & $\begin{array}{l}+2.52 \\
-4.95 \\
\end{array}$ \\
\hline Pointing $^{e}(\S 3.1)$ & -0.49 & $(-0.01)$ & $(-0.14)$ & -0.53 & -0.16 & -0.39 & $(-0.22)$ & $(+0.01)$ & $(+0.57)$ & -0.21 & -0.21 & -0.20 \\
\hline Image extent $^{e}(\S 3.2)$ & -2.55 & -2.00 & -3.82 & -2.91 & -2.32 & -2.64 & $(+0.19)$ & -0.46 & +2.37 & -0.34 & -0.35 & -0.29 \\
\hline Mirrors $^{e}(\S 3.3)$ & +2.13 & +4.11 & $(+0.45)$ & +4.74 & +4.76 & +4.91 & -5.51 & +3.69 & -2.21 & $(+0.01)$ & $(+0.09)$ & +0.19 \\
\hline $\mathrm{GV}$ (Be filter $)^{e}(\S 3.4)$ & $\begin{array}{l}+3.85 \\
-12.3\end{array}$ & $\begin{array}{l}(+0.06) \\
-3.53\end{array}$ & $\begin{array}{l}(+0.05) \\
-2.93\end{array}$ & $\begin{array}{l}+3.54 \\
-12.7\end{array}$ & $\begin{array}{l}+0.80 \\
-6.08\end{array}$ & $\begin{array}{l}+2.50 \\
-10.0\end{array}$ & $\begin{array}{l}+6.09 \\
-10.2\end{array}$ & $\begin{array}{l}(+0.13) \\
-1.34\end{array}$ & $\begin{array}{l}(+0.41) \\
(-0.11)\end{array}$ & $\begin{array}{l}+1.46 \\
-4.19\end{array}$ & $\begin{array}{l}+1.31 \\
-3.96\end{array}$ & $\begin{array}{l}+1.36 \\
-3.97\end{array}$ \\
\hline $\mathrm{GV}(\text { geom. })^{e}(\S 3.4)$ & - & - & - & - & - & - & -1.12 & +2.35 & +5.88 & +0.54 & +0.60 & +0.79 \\
\hline Aperture filters $^{e}(\S 3.5)$ & \pm 0.31 & \pm 0.13 & $( \pm 0.01)$ & \pm 0.34 & \pm 0.20 & \pm 0.29 & $( \pm 0.13)$ & $( \pm 0.06)$ & $( \pm 0.10)$ & $( \pm 0.08)$ & $( \pm 0.08)$ & $( \pm 0.08)$ \\
\hline Detector eff. $^{e}(\S 3.6)$ & $( \pm 0.01)$ & \pm 1.01 & \pm 3.32 & \pm 0.81 & \pm 0.35 & \pm 0.43 & $( \pm 0.01)$ & \pm 1.33 & $( \pm 0.62)$ & \pm 0.66 & \pm 0.68 & \pm 0.71 \\
\hline LSF tail $^{e}(\S 3.7)$ & \pm 0.53 & \pm 0.62 & \pm 7.12 & \pm 1.39 & \pm 0.31 & \pm 1.02 & \pm 1.38 & $( \pm 0.10)$ & \pm 5.20 & \pm 0.78 & \pm 0.48 & \pm 0.91 \\
\hline Energy gain ${ }^{e}(\S 3.8)$ & - & - & +1.30 & - & - & - & - & - & $(-0.90)$ & - & - & - \\
\hline Screening $^{e}(\S 3.9)$ & +3.24 & +4.56 & +20.6 & +1.54 & +3.02 & +1.74 & +2.09 & -3.77 & $(-0.15)$ & -1.06 & -1.38 & -1.65 \\
\hline $\mathrm{NXB}^{e}(\S 3.10)$ & $( \pm 0.01)$ & $( \pm 0.00)$ & $( \pm 0.37)$ & $( \pm 0.01)$ & $( \pm 0.00)$ & $( \pm 0.00)$ & $( \pm 0.01)$ & $( \pm 0.01)$ & $( \pm 0.58)$ & $( \pm 0.01)$ & $( \pm 0.00)$ & $( \pm 0.01)$ \\
\hline Spec model $^{e}(\S 3.11)$ & \pm 1.45 & \pm 0.17 & $( \pm 0.01)$ & \pm 1.50 & \pm 0.53 & \pm 1.13 & \pm 1.40 & \pm 0.17 & $( \pm 0.11)$ & \pm 0.54 & \pm 0.52 & \pm 0.52 \\
\hline \multirow[t]{2}{*}{ Goodness of fit } & \multicolumn{4}{|c|}{ Degree of freedom $f$} & 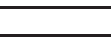 & - & \multicolumn{5}{|c|}{ Reduced $\chi^{2}$} & \\
\hline & 1997 & 7997 & 11997 & 11497 & 9997 & 23497 & $1.10-1.23$ & $1.02-1.05$ & $0.94-0.94$ & $1.08-1.57$ & $1.05-1.17$ & $1.02-1.31$ \\
\hline
\end{tabular}

${ }^{a}$ Absorption-corrected flux in the relevant energy band in the unit of $10^{-8} \mathrm{erg} \mathrm{s}^{-1} \mathrm{~cm}^{-2}$.

${ }^{b}$ Best-fit values of the fiducial model. ${ }^{c}$ The percentage of the $1 \sigma$ statistical uncertainty with respect to the best-fit value of the fiducial model.

${ }^{d}$ Quadrature sum of all the systematic uncertainty terms.

${ }^{e}$ For each cause of the systematic uncertainty, the best-fit values are evaluated. (Evaluated value - fiducial model value)/(fiducial model value) is shown in percentage. The values less than the statistical uncertainties indicate that the difference has no significance, which are shown in the parentheses.

${ }^{f}$ Goodness of fit by the degree of freedom and the ranges of the reduced $\chi^{2}$ values.

\subsection{X-ray mirror}

The effective area of the X-ray mirror assembly was measured on the ground at six energies of the continuum up to $\sim 20 \mathrm{keV}$ (Sato et al. 2016a; Iizuka et al. JATIS, submitted) and additional detailed measurements of the Au L (Kikuchi et al. 2016; Maeda et al. 2016) and M (Kurashima et al. 2016) edges. The Au L3 to L1 edges at $11.919,13.734$, and $14.353 \mathrm{keV}$ and the M5 to M1 edges at 2.206, 2.291, 2.743, 3.148, and $3.425 \mathrm{keV}$ are the most prominent features in the effective area curve of the Au-coated mirrors (figure 4f), which are also recognized in the actual spectrum (figure $4 a)$.

The mirrors are characterized by various quantities such as the reflectivity, surface roughness, degrees of misalignment, which are used by the ray-tracing simulator program to calculate the mirror effective area. Because of the limited quantity of ground calibration measurements and a large statistical uncertainty in the ray-tracing calculation at small effective areas, no reliable effective area curve can be obtained above $\sim 20 \mathrm{keV}$ (Yaqoob et al. JATIS, submitted). Below $\sim 20 \mathrm{keV}$, discrepancies between the ground-based effective area measurements and those from the ray-tracing simulator up to $\sim 10 \%$ are known. The discrepancy is much larger than the statistical uncertainty in the ray-tracing output below $\sim 10 \mathrm{keV}$, but they are comparable at $\sim 20 \mathrm{keV}$. The difference is interpolated to make a smooth correction curve called the "SXT-S fudge" factor (figure 4e). We assessed the level of systematic uncertainty by comparing the effective area curve with and without the fudge factor.

\subsection{Gate valve}

The gate valve has three components that affect the effective area: (1) a Be filter that allows some X-ray transmission, (2) a stainless steel protective screen, and (3) a cross structure made of a thick $\mathrm{Al}$ for mechanical support (Eckart et al. 2016).

(1) The Be filter has a thickness of $\sim 262 \mu \mathrm{m}$ and a density of $1.85 \mathrm{~g} \mathrm{~cm}^{-3}$ with a small amount of $\mathrm{Mn}, \mathrm{Fe}$, and $\mathrm{Ni}$ (Hoshino et al. 2017; Yoshida et al. 2017). The transmission curve of the filter is characterized by the photo-electric absorption by $\mathrm{Be}$ and other species, as well as the Bragg diffraction losses by Be. We made measurements of the flight spare window at ground synchrotron facilities after the launch, which were limited to energies above $2.5 \mathrm{keV}$. Among them, the measurements and the modeling below $3.8 \mathrm{keV}$ remain highly uncertain. (2) The stainless screen covers $29 \%$ of the geometrical area and it reduces the flux by the fraction independent of the energy, except for energies above $\sim 20 \mathrm{keV}$ where it becomes partially transmissive. (3) The thick Al support cross, which is opaque to $\mathrm{X}$-rays, is aligned with the mirror quadrant gaps but has a geometry such that it still blocks a significant fraction $(\sim 23.3 \%)$ of incident X-rays. The transmission curves of the Be filter and the stainless screen are shown in figure 4 (d).

There are two major sources of uncertainty. One is the Be filter transmission, which dominates the total effective area curve below $\sim 5 \mathrm{keV}$ (figure 3f). We changed the transmission within the measurement errors and evaluated its effect. The other is the geometrical effect by the support cross that partially inter- 
venes the X-rays far above the focal plane. As the higher energy X-rays are more preferentially reflected by the inner part of the nested foils with smaller incident angles, they are more likely to be blocked by the gate valve. The correction of this energy-dependent factor was implemented in the ARF generator assuming that the source is point-like (Yaqoob et al. JATIS, submitted). In theory, it depends on the source distribution. We assessed its systematic uncertainty by comparing the correction curve in two extreme cases that (i) the source is point-like and (ii) the source is uniform across the SXS field of view. The curves for the two cases are shown in figure 4 (e). The ARF is generated to match with the assumed spatial distribution, thus we can only compare the power-law index by this method.

\subsection{Aperture filters}

The SXS has a total of five filters in the X-ray light path. All five are made of an Aluminized polyimide thin film with thicknesses of approximately 150-200 nm ( 50-100 nm Al and $\sim 90$ $110 \mathrm{~nm}$ polyimide). They are anchored at different temperature stages from $50 \mathrm{mK}$ at the sensor thermal sink to $\sim 300 \mathrm{~K}$ at the Dewar main shell. The outer three filters are supported by $\mathrm{Si}$ meshes. The combined transmission is shown in figure 4 (d) with the prominent $\mathrm{Al}$ and small Si K edge features.

The combined filter transmission is calibrated much better than the required $5 \%$ at $0.5,1.5$, and $6 \mathrm{keV}$ (Eckart et al. 2016). We modified the thickness by $\pm 1 \%$ for the entire bandpass and estimated its effect.

\subsection{Detector efficiency}

The detector of the SXS consists of an array of $6 \times 6$ pixels made by $\mathrm{HgTe}$ of $\sim 10.5 \pm 0.1 \mu \mathrm{m}$ thickness. The quantum efficiency is $100 \%$ below the energy around the Te L1 edge at $4.949 \mathrm{keV}$, and monotonically decreases as the energy increases except for the $\mathrm{Hg}$ L3 to L1 edges at 12.284, 14.209, and $14.839 \mathrm{keV}$ (figure $4 \mathrm{~d})$. The detector filling fraction is $\sim 90 \%$.

The efficiency is calibrated better than the required $5 \%$ at $6 \mathrm{keV}$ (Eckart et al. 2016). We modified the absorber thickness by $\pm 5 \%$ for the entire bandpass from the best-fit value and estimated its effect.

\subsection{Extended LSF}

The detector response to monochromatic X-rays consists of (i) the main Gaussian peak of $\sim 5 \mathrm{eV}$ for high-quality grades, (ii) the exponential low-energy tail of an $e$-folding scale of $12 \mathrm{eV}$, (iii) the escape peaks by $\mathrm{Hg}$ and $\mathrm{Te}$, (iv) the electron loss continuum, and (v) the X-ray fluorescence of Si (Leutenegger et al. 2016; Eckart et al. 2016). For (i) and (ii), events are redistributed to the energies localized to the incident energy (hereafter, the "main" components). For (iii) and (iv), redistributed events are spread over a wide range of energies below the incident energy due to the energy loss either by escaping fluorescent X-rays or electrons (the "tail" components). For (v), the redistributed events are only significant at $\mathrm{K} \alpha(1.74 \mathrm{keV})$.

In figure 4 (a), we decomposed the observed spectrum into the main, tail, and Si fluorescence components. The tail component is larger than the main component below $\sim 1.8 \mathrm{keV}$ since the gate valve Be window blocks low-energy celestial photons from reaching the detector. The tail components are modeled using the fraction of the electron loss continuum and 51 escape peaks, which amounts to a few percent of the main peak in total. A detailed modeling is underway, but we currently have $\approx 50 \%$ uncertainty in these fractions (Leutenegger et al. 2016). We thus changed the fractions collectively by $\pm 50 \%$ by keeping the unity of the redistribution and evaluated its effect in the fitting and assessed the corresponding uncertainty.

\subsection{Energy gain}

The energy gain scale is non-linear for the SXS. The gain curve for each pixel was derived by fitting ground calibration data (4.5-13.5 keV) to a fourth order polynomial, providing an accuracy of $\pm 2 \mathrm{eV}$ across that bandpass (Eckart et al. 2016). These gain curves were adjusted for flight operating conditions using limited on-orbit data (primarily the ${ }^{55} \mathrm{Fe}$ data on the calibration pixel and a single exposure of the main array to calibration photons from the filter wheel ${ }^{55} \mathrm{Fe}$ source; Leutenegger et al. 2016). Above the bandpass, the deviation from the model increases, which alters the spectral shape of the model. It is estimated that the current gain is under-estimated by $45-100 \mathrm{eV}$ at $20 \mathrm{keV}$ (Eckart et al. JATIS submitted). Assuming that a gain shift of $-100 \mathrm{eV}$ at $20 \mathrm{keV}$, this changes the power-law index and the flux at $12-24 \mathrm{keV}$ by $-0.9 \%$ and $1.3 \%$ respectively.

\subsection{Event screening}

Events are screened using various flags in the pipeline processing. In the present observation, $3.8 \%$ of all recorded events were removed by this screening. The spectrum of the screened events is shown in figure 4 (a). There are two major types of flags; one is based on the pulse shape of events and the other is based on the time coincidence with other events. All screenings are intended to remove only spurious events, but some X-ray events are removed by false positives. This effectively reduces the exposure time and changes the spectral shape. Some false positives can be calculated. For example, the anti-co veto screening reduces the exposure time by $\sim 0.05 \%$ for removing events arriving within $\pm 0.5 \mathrm{~ms}$ of an anti-co event above $30 \mathrm{keV}$ at an average rate of $0.54 \mathrm{~s}^{-1}$ during the Crab observation. Others are more difficult to quantify.

One way to assess the degree of unintended loss of events is to compare the observed and theoretical incoming rate. The 


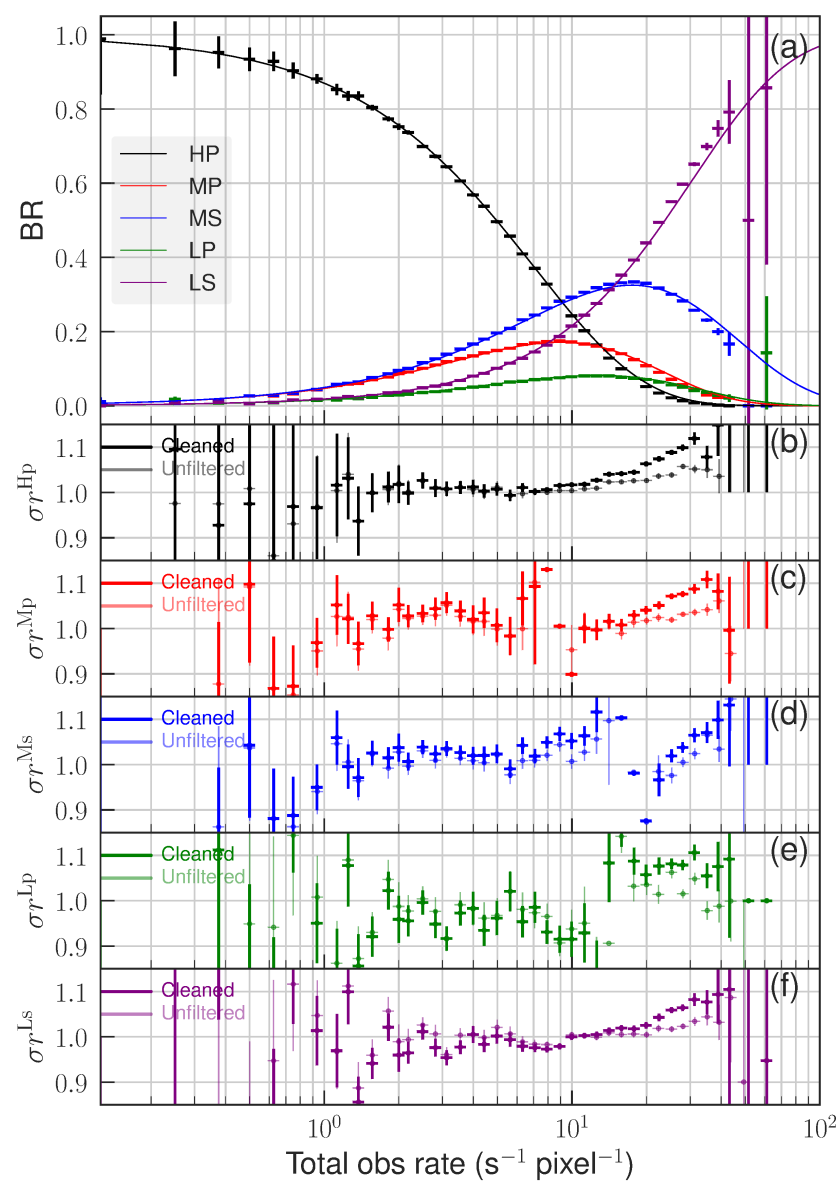

Fig. 6. (a) Observed branching ratio as a function of the observed total rate for the five grades (crosses) and the theoretical predictions assuming that the arrival time is random (solid curves). (b-f) For each grade, the ratio of the observed and theoretical total count rate is shown by using screened (dark) and unscreened (light color) events.

theoretical rate here is defined as an incoming rate that explains the observed grade branching ratio based on the Poisson statistics. Assume that the grade $g$ has a theoretical branching ratio of $P^{(g)}(r)$ at a total incoming rate of $r$ (figure 6a). We used the cleaned events of the entire Crab observation to derive the observed count rate of each grade $r_{\text {obs }}^{(g)}$ at every $8 \mathrm{~s}$ binning and grouped them by the total observed count rate defined by $r_{\mathrm{obs}}=\Sigma_{g} r_{\mathrm{obs}}^{(g)}$. The binning is sufficiently large in comparison to the pulse period, so that the arrival times can be considered to follow the Poisson statistics. Here, we corrected the rates for the intensity-dependent pixel dead times. By using all pixels, a wide range of $r_{\mathrm{obs}}^{(g)}$ can be covered. For each group of an $r_{\mathrm{obs}}$ value, the total theoretical count rate $r_{\text {th }}^{(g)}$ was derived so that it satisfies $P^{(g)}\left(r_{\mathrm{th}}^{(g)}\right)=r_{\mathrm{obs}}^{(g)} / r_{\text {obs }}$ for each grade $g$. The deviation is defined as $\sigma r^{(g)}=r_{\mathrm{th}}^{(g)} / r_{\mathrm{obs}}^{(g)}$ (figure 6b-f).

The $\sigma r^{(g)}$ value is consistent with 1.0 at count rates lower than $10 \mathrm{~s}^{-1}$ pixel $^{-1}$, indicating that the loss by event screening is negligible. It increases above 1.0 significantly at a higher count rate up to $\sim 10 \%$, suggesting that some X-ray counts are lost. At least a half of them can be explained by screening. Indeed, if we perform the same exercise using the unscreened events, the discrepancy decreases by about a half as shown in light colors in figure 6 (b)-(f). If two events of the same pixel are too close in time, they are not even distinguished as two events. This effect is not large, however, as we see no trend of grade migration from Ls or Lp to Hp.

$\mathrm{X}$-ray events with higher energies are more prone to inappropriate flagging as they have a longer pulse shape. Because of this, the spectrum can be distorted by unintended screening. We evaluated this effect by comparing the fitting results of the spectra made with unscreened or screened events.

\subsection{NXB}

The NXB spectrum was constructed by integrating the data during Earth pointing and weighting by the history of the Earth magnetic cut-off rigidity. The spectrum is flat over the entire energy band (figure 4a) with some features of $\mathrm{Mn} \mathrm{K} \alpha$ by scattered X-rays from the ${ }^{55} \mathrm{Fe}$ calibration source and $\mathrm{Au} \mathrm{L} \alpha$ and $\mathrm{L} \beta$ by fluorescence $\mathrm{X}$-rays primarily from the Au layer of the sensor array frame (Kilbourne et al. 2016b).

We assumed that the spectrum above $30 \mathrm{keV}$ is dominated by the NXB. The systematic uncertainty of the NXB was evaluated by rescaling the NXB spectrum within the $1 \sigma$ Poisson fluctuation of the counts above $30 \mathrm{keV}$. The NXB spectra were generated for the minimum and maximum rescaling factors $(0.56$ and 1.74. respectively), which were subtracted for the fitting to evaluate their systematic uncertainties. As the NXB is almost negligible, there are no significant systematic uncertainties except for the high energy band.

\subsection{Spectral model}

Because the SXS data has little constraining power for the amount of interstellar extinction due to its low effective area below $\sim 2 \mathrm{keV}$, we applied the extinction model that best describes the Chandra LETG spectrum (Weisskopf et al. 2004). However, the LETG result is not exactly applicable to the SXS spectrum primarily because the event extraction regions and the PSF are not the same. The extinction column is known to vary across the nebula (Mori et al. 2004).

We evaluated how our fitting result is affected by changing the extinction column values over $(3.26-5.64) \times 10^{21} \mathrm{~cm}^{-2}$ (figure $4 \mathrm{~g}$ ), which are two extreme values in a cross-calibration study (Kirsch et al. 2005). 


\section{Discussion}

\subsection{Major causes for the residuals}

We now assessed the effects of the systematic uncertainty by various conceivable causes (table 3). Different causes leave a prominent effect in different energy bands. We attempt to identify the primary causes for these residuals below.

First, for each one of the causes, we identified the energy band in which the cause can change the spectral shape without changing the shape in other bands. We grouped the causes into two: the "additive" causes and the "multiplicative" causes. The former consists of the RMF tail ( $\S 3.7)$, the event screening (§3.9), and the NXB ( $\S 3.10)$, and the latter consists of the others. For the additive causes, the energy band was selected in which the additive spectrum exceeds $10 \%$ of the main component of the source spectrum (figure 4a). For the multiplicative causes, the transmission, efficiency, or the correction factor (figure $4 \mathrm{~d}, \mathrm{e}, \mathrm{f}$, and $\mathrm{g}$ ) as a function of energy $f(E)$ is used to calculate $|d f(E) / d E|$ at logarithmically-equally spaced energies at $0.5-30 \mathrm{keV}$. Then, a $20 \%$ of the energies were selected from the top among those sorted with a decreasing order of $|d f(E) / d E|$. The energy bands are shown in figure 4 (c).

The residual below $\sim 2 \mathrm{keV}$ is attributable to the LSF tail (§ 3.7) or the event screening ( 3.9 ), which is larger than the other additive cause and the main component of the spectrum (figure 4a). If we attribute the residual entirely to the LSF tail, a $\sim 20 \%$ increased value of the tail fraction gives the smallest residual. The residual at $\sim 2-4 \mathrm{keV}$ is attributable to the gate valve Be filter ( $\$ 3.4$ ) or the mirror effective area around the $\mathrm{Au} \mathrm{M}$ edges (§ 3.3). The residual at $\sim 8-16 \mathrm{keV}$ is attributable to the mirror effective area $(\S 3.3)$, the event screening ( $\$ 3.9)$, the geometrical effect of the gate valve ( $\S 3.4)$, or the detector efficiency ( $\S 3.6$ ). They are hard to be decoupled, but their fractional contribution can be assessed in the 4-12 keV fitting result in table 3 , in which the mirror effective area and the event screening are the largest. It is illustrated by the change in the residual ratio from $<1$ to $>1$ (figure 4 b) at the Au L3 edge attributable to the mirrors, but not at the $\mathrm{Hg} \mathrm{L} 3$ edge attributable to the detector efficiency. Together with the Au M features, the Au L features of the mirror effective area is not easy to calibrate and model. A typical systematic uncertainty around theses edges is estimated to be $\approx 10 \%$ (Kurashima et al. 2016).

Given the limited amount of in-flight data, it is difficult to disentangle these causes further. For the other objects, multiplicative causes can be corrected by applying the Crab ratio, which is defined as the ratio between the SXS spectrum and the canonical model presented here ${ }^{3}$.

Above $\sim 20 \mathrm{keV}$, the SXS is not very well calibrated, especially because of the lack of measurement and hence modeling

\footnotetext{
${ }^{3}$ We provide the Crab ratio correction factor file, which is available upon request.
}

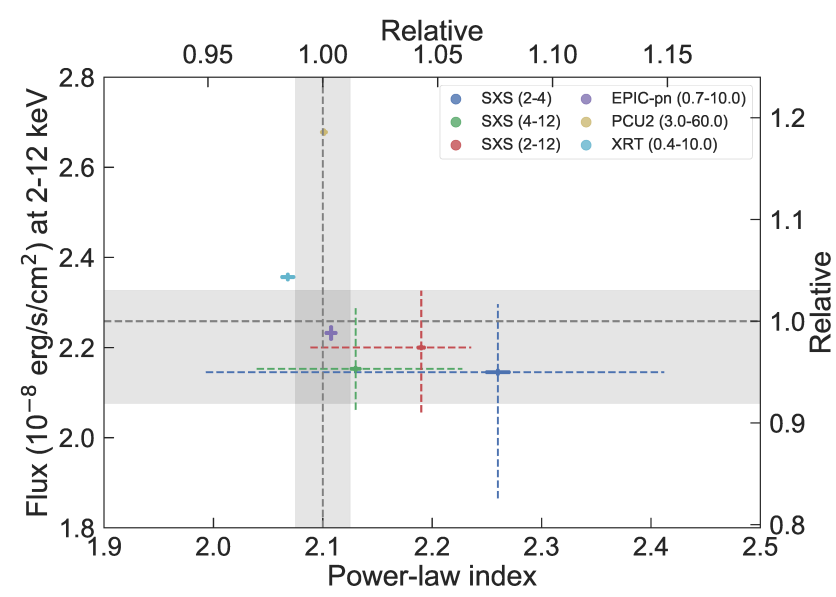

Fig. 7. Comparison between the SXS and the others (Weisskopf et al. 2010; Kirsch et al. 2005) for the flux and the power-law index. The data are obtained by fitting in different energy bands, which is shown in the parentheses in the legend in the unit of $\mathrm{keV}$. The flux values are converted to that in the 2-12 keV band, which is corrected for the absorption for this plot. For SXS, thick solid error bars are for the statistical errors, while thin dashed bars are for the quadrature sum of the systematic errors (table 3). Dashed gray lines indicate the values for the IACHEC model, whereas the shaded gray indicates their typical range of the intrinsic variation.

of the mirrors due primarily to the low mirror effective area at high energies. Such high-energy bands are far beyond our requirement. Although we have a clear signal above the NXB above $20 \mathrm{keV}$, the data cannot be used for scientific purposes.

\subsection{Comparison with IACHEC results}

We compare our results with some preceding work using different instruments. In figure 7, we show the best-fit values of the power-law index and the $2-12 \mathrm{keV}$ flux of the SXS (table 3) as well as those of XMM-Newton EPIC-pn, RXTE PCU2 (Weisskopf et al. 2010), and Swift XRT (Kirsch et al. 2005). The statistical errors are shown for the SXS and the other data, while the quadrature sum of all the systematic errors (table 3) are shown for the SXS data.

The IACHEC model, in which all the parameters are fixed to those in Weisskopf et al. (2010), is shown in gray dashed lines. The Crab spectrum is known to be variable both in the flux and the power-law index (Wilson-Hodge et al. 2011). We derived the typical variation of the flux using the monitoring data with the Monitor of All-sky X-ray Image (MAXI; Matsuoka et al. 2009) and Gamma-ray Burst Monitor (GBM; Meegan et al. 2009) onboard the Fermi observatory, and that of the powerlaw index using RXTE PCA (Shaposhnikov et al. 2012). The intrinsic variation is indicated with gray shades.

For the flux, the best-fit value in the fiducial model of the SXS is consistent with the IACHEC model. On the other hand, that of the power-law index for the SXS is softer than the IACHEC model and also with the other data. This is not due 
to the small extraction region of the SXS. The softer index is consistently seen in all phase-resolved spectroscopy (figure 5b), in which the contrast of the pulsar and the nebular component changes. Also, when the extraction region is smaller, we would expect that the index becomes harder (Madsen et al. 2015b) as the fraction of the harder pulsar component increases. The statistical uncertainty is negligible. Therefore, it is likely that the discrepancy is due to the systematic uncertainty of the SXS. In fact, the systematic uncertainty of the power-law index, which is primarily due to the gate valve and event screening calibrations, is large enough to explain most of the discrepancy between the SXS result and the IACHEC model.

\section{Summary}

We presented the result of the in-flight calibration of the effective area of the SXS using the Crab observations. The $0.5-$ $20 \mathrm{keV}$ spectrum was described by a single power-law continuum attenuated by the interstellar extinction. We evaluated the level of systematic uncertainty associated with various calibration items. A quadrature sum of these uncertainties amounts to about $+6 /-7 \%$ and $+2 /-5 \%$ respectively for the flux and the power-law index in the $2-12 \mathrm{keV}$ band. The best-fit value of the flux with the SXS is consistent with the others, whereas that of the power-law index is softer. However, the discrepancy is mostly accountable within the systematic uncertainty of the SXS. The primary causes of the softer spectrum of the SXS than the others are the calibration of the gate valve transmission and the event screening.

\section{Acknowledgments}

The authors gratefully acknowledge the many other scientists, engineers, technicians, and students whose hard work contributed to the capabilities of the SXS. We also appreciate Yuki Yoshida and Shunji Kitamoto at Rikkyo University for providing the spare Be filter transmission data. This work was supported by JSPS KAKENHI Grant Numbers JP15H03642, JP16K05309, JP25105516, JP23540280, and the Grant-inAid for Scientific Research on Innovative Areas Nuclear matter in neutron stars investigated by experiments and astronomical observations. T. S. is supported by the Research Fellow of JSPS for Young Scientists.

\section{References}

Angelini, L., Terada, Y., Loewenstein, M., et al. 2016, in Proc. SPIE, Vol. 9905, Society of Photo-Optical Instrumentation Engineers (SPIE) Conference Series, 990514

Arnaud, K. A. 1996, in Astronomical Society of the Pacific Conference Series, Vol. 101, Astronomical Data Analysis Software and Systems V, ed. G. H. Jacoby \& J. Barnes, 17

Eckart, M. E., Adams, J. S., Boyce, K. R., et al. 2016, in Proc. SPIE, Vol. 9905, Society of Photo-Optical Instrumentation Engineers (SPIE) Conference Series, 99053W
Hayashi, T., Sato, T., Kikuchi, N., et al. 2016, in Proc. SPIE, Vol. 9905, Space Telescopes and Instrumentation 2016: Ultraviolet to Gamma Ray, 99055D

Hitomi Collaboration. 2018, PASJ

Hoshino, A., Yoshida, Y., Kitamoto, S., et al. 2017, in Society of PhotoOptical Instrumentation Engineers (SPIE) Conference Series, Vol. 10397, Society of Photo-Optical Instrumentation Engineers (SPIE) Conference Series, 103970E

Iizuka, R., Hayashi, T., Maeda, Y., et al. 2014, in Proc. SPIE, Vol. 9144, Space Telescopes and Instrumentation 2014: Ultraviolet to Gamma Ray, 914458

Ishisaki, Y., Yamada, S., Seta, H., et al. 2016, in Proc. SPIE, Vol. 9905, Society of Photo-Optical Instrumentation Engineers (SPIE) Conference Series, 99053T

Kaastra, J. S., de Vries, C. P., Costantini, E., \& den Herder, J. W. A. 2009, A\&A, 497, 291

Kelley, R. L., Akamatsu, H., Azzarello, P., et al. 2016, in Proc. SPIE, Vol. 9905, Society of Photo-Optical Instrumentation Engineers (SPIE) Conference Series, 99050V

Kikuchi, N., Kurashima, S., Ishida, M., et al. 2016, Optics Express, 24, 25548

Kilbourne, C. A., Adams, J. S., Arsenovic, P., et al. 2016a, in Proc. SPIE, Vol. 9905, Society of Photo-Optical Instrumentation Engineers (SPIE) Conference Series, 99053Q

Kilbourne, C. A., Adams, J. S., Brekosky, R. P., et al. 2016b, in Proc. SPIE, Vol. 9905, Society of Photo-Optical Instrumentation Engineers (SPIE) Conference Series, 99053L

Kilbourne, C. A., Sawada, M., Tsujimoto, M., et al. 2018, PASJ

Kirsch, M. G., Briel, U. G., Burrows, D., et al. 2005, in Proc. SPIE, Vol. 5898, UV, X-Ray, and Gamma-Ray Space Instrumentation for Astronomy XIV, ed. O. H. W. Siegmund, 22-33

Kurashima, S., Furuzawa, A., Sato, T., et al. 2016, in Proc. SPIE, Vol. 9905, Space Telescopes and Instrumentation 2016: Ultraviolet to Gamma Ray, 99053Y

Leutenegger, M. A., Audard, M., Boyce, K. R., et al. 2016, in Proc. SPIE, Vol. 9905, Society of Photo-Optical Instrumentation Engineers (SPIE) Conference Series, 99053U

Lobanov, A. P., Horns, D., \& Muxlow, T. W. B. 2011, A\&A, 533, A10

Madsen, K. K., Harrison, F. A., Markwardt, C. B., et al. 2015a, ApJS, 220,8

Madsen, K. K., Reynolds, S., Harrison, F., et al. 2015b, ApJ, 801, 66

Maeda, Y., Kikuchi, N., Kurashima, S., et al. 2016, in Proc. SPIE, Vol. 9905, Space Telescopes and Instrumentation 2016: Ultraviolet to Gamma Ray, 99053Z

Maeda, Y., Sato, T., Hayashi, T., et al. 2018, PASJ

Matsuoka, M., Kawasaki, K., Ueno, S., et al. 2009, PASJ, 61, 999

Meegan, C., Lichti, G., Bhat, P. N., et al. 2009, ApJ, 702, 791

Mori, K., Burrows, D. N., Hester, J. J., et al. 2004, ApJ, 609, 186

Okajima, T., Soong, Y., Serlemitsos, P., et al. 2016, in Proc. SPIE, Vol. 9905, Society of Photo-Optical Instrumentation Engineers (SPIE) Conference Series, $99050 \mathrm{Z}$

Porter, F. S., Boyce, K. R., Chiao, M. P., et al. 2016, in Proc. SPIE, Vol. 9905, Society of Photo-Optical Instrumentation Engineers (SPIE) Conference Series, 99050W

Sato, T., Iizuka, R., Hayashi, T., et al. 2014, in Proc. SPIE, Vol. 9144, Space Telescopes and Instrumentation 2014: Ultraviolet to Gamma Ray, 914459 
Sato, T., Iizuka, R., Ishida, M., et al. 2016a, Journal of Astronomical Telescopes, Instruments, and Systems, 2, 044001

Sato, T., Iizuka, R., Mori, H., et al. 2016b, in Proc. SPIE, Vol. 9905, Space Telescopes and Instrumentation 2016: Ultraviolet to Gamma Ray, 99053X

Shaposhnikov, N., Jahoda, K., Markwardt, C., Swank, J., \& Strohmayer, T. 2012, ApJ, 757, 159

Takahashi, T., Kokubun, M., Mitsuda, K., \& et al. 2016, in Proc. SPIE, Vol. 9905, Society of Photo-Optical Instrumentation Engineers (SPIE) Conference Series, 99050U

Tsujimoto, M., Mitsuda, K., Kelley, R. L., et al. 2016, in Proc. SPIE, Vol. 9905, Society of Photo-Optical Instrumentation Engineers (SPIE) Conference Series, 99050 Y

Weisskopf, M. C., Guainazzi, M., Jahoda, K., et al. 2010, ApJ, 713, 912

Weisskopf, M. C., O’Dell, S. L., Paerels, F., et al. 2004, ApJ, 601, 1050

Willingale, R., Aschenbach, B., Griffiths, R. G., et al. 2001, A\&A, 365, L212

Wilms, J., Allen, A., \& McCray, R. 2000, ApJ, 542, 914

Wilson-Hodge, C. A., Cherry, M. L., Case, G. L., et al. 2011, ApJL, 727, L40

Yoshida, Y., Hoshino, A., Kitamoto, S., et al. 2017, in Society of PhotoOptical Instrumentation Engineers (SPIE) Conference Series, Vol. 10397, Society of Photo-Optical Instrumentation Engineers (SPIE) Conference Series, 103971D 